\title{
Criminologie
}

\section{Transformations récentes de la législation fédérale sur la mise en liberté sous condition au Canada. Une lecture à la lumière des écrits sur la notion de risque}

\section{Dominique Robert}

Volume 34, numéro 1, printemps 2001

La notion de risque dans la gestion pénale

URI : https://id.erudit.org/iderudit/004758ar

DOI : https://doi.org/10.7202/004758ar

Aller au sommaire du numéro

Éditeur(s)

Les Presses de l'Université de Montréal

ISSN

0316-0041 (imprimé)

1492-1367 (numérique)

Découvrir la revue

Citer cet article

Robert, D. (2001). Transformations récentes de la législation fédérale sur la mise en liberté sous condition au Canada. Une lecture à la lumière des écrits sur la notion de risque. Criminologie, 34(1), 73-99.

https://doi.org/10.7202/004758ar

\section{Résumé de l'article}

Cet article s'intéresse aux tendances de la loi sur la mise en liberté sous condition au Canada depuis le milieu des année 70. Contrairement à la conclusion selon laquelle la mise en liberté sous condition serait victime d'attrition, il est démontré que ce qui anime cette loi est davantage la tendance duale, aussi appelée bifurcation. De plus, il est avancé que cette tendance duale opère de plus en plus sur la base du critère de risque. La démonstration procède par l'examen des transformations du texte de la loi sur la mise en liberté sous condition depuis son implantation en 1899. L'observation des discours officiel et politique qui ont court à deux périodes charnières de cette mesure, 1956-1960 et 1988-1992, permet ensuite de voir comment ces discours stimulent la tendance duale et l'approche de gestion des risques observée dans la loi. 


\title{
Transformations récentes de la législation fédérale sur la mise en liberté sous condition au Canada. Une lecture à la lumière des écrits sur la notion de risque ${ }^{1}$
}

\author{
Dominique Robert \\ Étudiante au doctorat \\ École de criminologie \\ Université de Montréal \\ robertdo@magellan.umontreal.ca
}

RÉsumé - Cet article s'intéresse aux tendances de la loi sur la mise en liberté sous condition au Canada depuis le milieu des année 70. Contrairement à la conclusion selon laquelle la mise en liberté sous condition serait victime d'attrition, il est démontré que ce qui anime cette loi est davantage la tendance duale, aussi appelée bifurcation. De plus, il est avancé que cette tendance duale opère de plus en plus sur la base du critère de risque. La démonstration procède par l'examen des transformations du texte de la loi sur la mise en liberté sous condition depuis son implantation en 1899. L'observation des discours officiel et politique qui ont court à deux périodes charnières de cette mesure, 1956-1960 et 1988-1992, permet ensuite de voir comment ces discours stimulent la tendance duale et l'approche de gestion des risques observée dans la loi.

ABSTRACT - Beyond the conclusion stating the attrition of parole, this article wants to show that the trend canadian federal parole law has been experiencing since the mid- 70 's

1. Cet article est issu d'une réflexion faite à partir de mon mémoire de maittrise (1994) réalisé grâce à une bourse du Fonds pour la Formation de Chercheurs et l'Aide à la Recherche (FCAR) et grâce à l'appui du Groupe de recherche sur les pratiques et politiques pénales (GRAPPP). Je tiens à remercier Pierre Landreville, Marie Robert et Bruno Théorêt pour la lecture attentive qu'ils ont faite des différentes versions de ce texte et pour le temps consacré à la discussion ainsi que les évaluateurs qui m’ont obligée à préciser ma pensée. 
is one of twin track policy also known as bifurcation. The author also suggests that this orientation operates more and more under the risk criteria. The demonstration proceeds first by establishing the history of canadian parole law since its inception in 1899 to the present. Secondly, by analyzing more closely political and official discourses running through two historical periods, 1956-1960 and 1988-1992, it is shown that the background discourse of the law stimulates the trend toward bifurcation and the risk management approach.

\section{Introduction}

Au Canada, à chaque année depuis bientôt cinquante ans ${ }^{2}$, la très grande majorité des infracteurs qui sortent des pénitenciers le font selon les dispositions du régime fédéral de mise en liberté sous condition. En 1990, un regard porté sur les dernières publications officielles du ministère du Solliciteur général du Canada, les récents rapports des comités officiels et les écrits de la Commission nationale des libérations conditionnelles (CNLC) pouvait laisser croire que la mise en liberté sous condition, s'atrophiant progressivement, tendait inévitablement vers sa disparition (Brodeur, 1990). Chaque modification législative venait, semble-t-il, ajouter des conditions supplémentaires aux modalités de libération ou encore accroître la période d'inadmissibilité des détenus fédéraux à une forme de libération sous surveillance avant la fin de leur terme.

Les années écoulées depuis, et un retour sur la législation, permettent de croire que l'attrition ne résume pas, à elle seule, l'orientation que connaît actuellement la politique fédérale de mise en liberté sous condition au Canada. Dans le cadre de cet article, une tentative sera faite pour démontrer que la préoccupation pour la gestion du risque prend une ampleur nouvelle au sein de cette politique et nourrit la tendance duale ${ }^{3}$ qui s'y profile. En effet, depuis 1978, la politique de mise en liberté sous condition est de plus en plus à l'image d'une fourche à deux branches dont l'une indique la voie tracée pour les infracteurs « courants » alors que la seconde montre un autre chemin, celui adressé aux infracteurs dits violents ou dangereux.

2. Bien que la mise en liberté sous condition existe au Canada depuis 1899, ce n'est véritablement qu'avec l'instauration de la Commission nationale des libérations conditionnelles, en 1959, que la mesure se développe.

3. Le terme bifurcation est traduit ici par l'expression tendance duale. L'expression tendance duale renvoie simplement ici à l'image de la séparation, en deux parties, de ce qui était autrefois unitaire. 
Afin de soutenir les affirmations avancées ici, le point sera fait tout d'abord sur des écrits touchant la question de la gestion du risque dans le domaine correctionnel ainsi que la façon dont la tendance duale s'inscrit dans ce courant. La deuxième section démontrera ensuite empiriquement les transformations observées dans l'histoire de la politique de mise en liberté sous condition. C'est sur la description des changements législatifs concernant les modalités et la période d'inadmissibilité à la mise en liberté sous condition depuis son instauration, en 1899, que s'appuie le constat de la tendance duale.

Les troisième et quatrième sections de l'article cherchent à illustrer le fond idéel dans lequel émerge cette twin track policy. La démonstration procédera en comparant le discours politique avec le discours officiel ayant eu cours à deux périodes charnières pour la mise en liberté sous condition : les années 1956 à 1960 qui correspondent à la mise sur pied de la Commission nationale des libérations conditionnelles et les années 1988 à 1992 qui renvoient à l'adoption de la Loi sur le système correctionnel et la mise en liberté sous condition de 1992. Le « discours politique » dont il est question ici correspond en fait aux propos tenus par les élus à la Chambre des communes et au Comité permanent ${ }^{4}$. Le discours officiel renvoie quant à lui aux rapports des comités officiels qui ont été déposés à l'intérieur des périodes à l'étude soit le rapport Fauteux (1956) et le rapport Daubney (1988). Bien que ces types de matériau aient des limites certaines ${ }^{5}$, c'est à travers eux qu'on apprend les changements proposés par les émetteurs de la parole politique, mais surtout qu'on peut repérer le rationnel mis de l'avant pour produire un effet ainsi que les représentations

4. Pour la période 1956 à 1960, le Comité permanent de la justice et du solliciteur général n'est pas encore créé. C'est la Chambre elle-même qui se transforme en comité lors de la deuxième lecture, afin de discuter, article par article, des projets de loi sur les affaires correctionnelles.

5. Ce type de matériau est le résultat d'une enquête administrative visant à cerner un problème que présente une institution gouvernementale et à y apporter des solutions. Certains parlent des comités officiels comme de "pratiques d'impasse" (Brodeur, 1978) ou encore comme des façons de donner au peuple l'impression du changement politique et social (Normandeau, 1979). Certains auteurs ont éveillé notre méfiance envers de tels comités en démontrant que le postulat démocratique à la base de cette pratique est très souvent bafoué (Blumer, 1980 ; Robin, 1987). Malgré l'effet de façade, le manque de retombées et la partialité possible de ces comités, le rapport qui en est issu demeure digne d'intérêt dans la mesure où il crée une sorte d'effervescence politique, un espace où le changement est possible. En effet, les rapports de comités officiels ont très souvent des effets catalytiques (Chapman, 1973). À défaut de voir leurs recommandations se concrétiser en changements législatifs, les comités créent un momentum et lancent un signal à la bureaucratie qui doit commencer à penser et à mettre en place une réforme. 
qu'il suppose et promeut $^{6}$. On verra ainsi que la préoccupation pour le risque se dessine dans le discours et comment elle nourrit la tendance duale dans la politique de mise en liberté sous condition au Canada.

\section{Des études sur la gestion du risque}

Des études récentes qualifient les tendances correctionnelles contemporaines, et le contexte dans lequel elles prennent place, en terme de modèle assurantiel (Reichman, 1986), société du risque (Simon, 1987 ; O'Malley, 1992), modèle de contrôle social (Peters, 1986), pratique ou justice actuarielle (Simon, 1988 ; Feeley et Simon, 1994), ordre postdisciplinaire (Castel, 1991), technologie de gestion du risque (Ewald, 1991 ; Pratt, 1995), prudentialisme (O’Malley, 1992) ou encore en terme de nouvelle pénologie (Feeley et Simon, 1992 ; Simon et Feeley, $1995)^{7}$. Bien que ces expressions ne soient pas équivalentes, elles renvoient quand même toutes à une transformation qui opère dans le système pénal et qui fait une place de plus en plus grande à la logique assurantielle au sein de laquelle trône la notion de risque.

\section{Le modèle assurantiel de contrôle du crime et des infracteurs}

Reichman (1986) observe que, dans le secteur de la prévention de la criminalité, on assiste à l'utilisation croissante des concepts et des techniques

6. Les discours des députés à la Chambre des communes et lors des audiences du Comité permanent sont deux types de matériaux différents, l'un polémique, l'autre consensuel. Les débats en Chambre sont l'occasion pour les députés des différents partis de demander des comptes, de confronter l'adversaire sur les décisions prises ou qui tardent à se prendre, de démontrer au public, et aux autres membres du Parlement surtout, leur connaissance des enjeux et d'augmenter ainsi leur crédibilité. Les échanges lors des audiences du Comité permanent sont quant à eux le moment pour les députés de s'informer et d'en arriver à un compromis sur les amendements à apporter aux projets de lois. On reconnaît toutefois que la Chambre des communes et le Comité permanent ne sont pas le haut lieu des prises de décisions. En effet, la centralisation des pouvoirs dans les mains du gouvernement, l'inexpérience des députés (Franks, 1987) et le manque de participation aux échanges amènent à relativiser l'importance de ces discours par rapport à celui du comité exécutif par exemple.

7. Les différences dans le vocabulaire choisi pour caractériser l'état des politiques pénales ne sont pas fortuites. Certaines études embrassant un champ plus vaste que d'autres utilisent le terme de gestion du risque, plutôt que le concept de nouvelle pénologie par exemple, parce que plus général. Voir la note en fin de document 3 à la page 269 dans O'Malley (1992) pour une discussion plus approfondie sur la terminologie. 
propres à la logique assurantielle afin de gérer certaines possibilités d'infractions ou certains crimes avérés. Il semble en effet que le crime soit maintenant conçu au même titre qu'un accident de la route, c'est-à-dire comme une contingence que l'on tente de prévoir et dont on essaie de minimiser les impacts. Pour ce faire, on utilise divers moyens comme le durcissement de la cible (target hardening), l'établissement de profil (profiling), la réduction des opportunités, la prévention des pertes, etc.

Au-delà de la prévention de la criminalité, le modèle assurantiel a aussi été repéré dans le secteur correctionnel. Feeley et Simon $(1992,1994)$ désignent sous le terme de "nouvelle pénologie » cet ensemble de discours et de pratiques, en rupture avec la pénologie moderne, au sein duquel on ne cherche plus à transformer les individus mais plutôt à gérer les risques qu'ils représentent et le système lui-même. La nouvelle pénologie sous-tend et entretient une conception dé-moralisée du crime, c'està-dire une représentation de l'infraction qui n'est plus conçue comme une déviance antisociale mais plutôt comme un fait inévitable. On ne cherche donc plus à éliminer le crime mais plutôt à le gérer de façon à le rendre tolérable. Il s'agit en fait de minimiser les pertes, de diminuer les opportunités d'infractions et non de transformer les infracteurs ou la société en profondeur. Dans ce modèle, la responsabilité individuelle se dissout dans la socialisation des risques. Il s'ensuit un passage de l'obsession correctionnaliste, normalisatrice, à une préoccupation pour la gestion et à une utopie de la prédétection. Il y a déplacement du regard sur les causes du crime vers ses effets, du passé vers l'avenir.

Allant de pair avec la logique assurantielle, il y a les techniques actuarielles. D'après Feeley et Simon en effet, la criminologie clinique s'efface devant la description et la prédiction statistiques. Ces méthodes opèrent ainsi le fractionnement du sujet, c'est-à-dire la substitution, par une série de facteurs de risques, de ce qui était autrefois vu comme une «identité criminelle ». Les individus sont décomposés en variables et ces dernières sont corrélées et regroupées pour former des profils gradés selon le niveau de risque. L'unité de gestion passe de l'individu aux groupes. On cherche à les catégoriser, les classifier, les hiérarchiser. Des trajectoires institutionnelles sont tracées pour chaque groupe conformément au danger qu'il représente. Le diagnostic et le traitement ont fait place respectivement à l'attribution d'un profil et à l'assignation administrative ${ }^{8}$.

8. En fait, ces opérations de classification deviennent l'essence de l'entreprise correctionnelle : "Rather than simply extending the capacity of the system to rehabilitate or control crime, actuarial 
Le modèle prudentiel de contrôle du crime et la responsabilisation croissante

Si les changements qui s'opèrent dans le contrôle du crime et le secteur correctionnel peuvent être saisis, en partie, par la lunette du modèle assurantiel tel que le proposent Reichman (1986) et Feeley et Simon (1992 ;1994), O'Malley (1992) y voit surtout les effets du prudentialisme. Selon cette lecture des changements récents, la primauté est accordée, comme dans le modèle assurantiel, à la gestion des risques et à la prévention, mais la responsabilité, au lieu d'être socialisée comme dans le modèle assurantiel, est redistribuée aux individus, dans le cas présent, aux victimes potentielles et aux infracteurs avérés. Ce que le prudentialisme permet, donc, c'est de superposer la notion de responsabilité individuelle au système de gestion du risque. Ce faisant, la gestion des risques dans le domaine correctionnel est couplée à un mouvement de responsabilisation et de punitivité marquée à l'endroit des infracteurs à hauts risques (O'Malley, 1992 : 261).

Dans cette perspective, l'individu est l'bomo æconomicus (O'Malley, 1992 : 264). Les personnes sont donc perçues comme des agents libres de commettre ou non une infraction. « As Foucault made clear, what he saw as the "criminological labyrinth» was constructed around the assumption that crime is caused, and that cause reduces responsability (1977 : 252)» (O’Malley, 1992 : 265). Avec le modèle prudentiel et son homme rationnel, le regard sur les causes du crime est mis à l'écart, la responsabilité de l'individu est rehaussée et l'effet qui s'ensuit est une plus grande sévérité des sanctions.

Bref, la thèse de O'Malley, conjuguée à celles des tenants du modèle assurantiel, permet de saisir les changements qui se produisent dans le système correctionnel et qui pourraient paraître antinomiques, à savoir la place prépondérante accordée à la logique assurantielle et à la gestion du risque parallèlement à la responsabilisation des infracteurs.

classification has come increasingly to define the correctional entreprise itself" (Feeley et Simon, 1992 : 454). Peters abonde dans le même sens et remarque que la tendance qui s'impose maintenant en pénologie relève du paradigme de l'« ingénierie sociale » (1986:31). Les objectifs du système correctionnel se retrouvent isolés des fins sociales qui servaient autrefois à mesurer son efficacité. Par exemple, le retour en incarcération suite à la récidive d'un individu libéré conditionnellement n'est plus vu comme un échec du processus pénal mais plutôt comme une preuve que le système parvient à se contrôler lui-même, à réagir à ses ratés. 


\section{La gestion des risques et la tendance duale}

C'est à la fin des années 70 que Bottoms ${ }^{9}$ identifie la tendance duale dans la gestion correctionnelle de la dangerosité. Une décennie plus tard, des analystes des politiques correctionnelles canadiennes repèrent aussi ce mouvement (Ericson, 1987) ${ }^{10}$. Cavadino et Dignan définissent ainsi cette tendance :

Bifurcation refers to a dual-edged approach to the problem of offending: differentiating between "ordinary" or "run of the mill" offenders with whom less severe measure can be taken on the one hand and, on the other hand "exceptional', "very serious" or "dangerous offenders" who can be made subject to much tougher measures (Cavadino et Dignan, $1992: 23$ ).

La tendance duale réfere donc au fait de punir de plus en plus sévèrement les « vrais » infracteurs, les « dangereux », alors qu'à l'inverse, les "petits» infracteurs sont traités avec une souplesse croissante. Que l'opération de classification produise inévitablement un fossé entre des catégories opposées de déviants, ceux construits comme ayant besoin d'un contrôle sévère et ceux construits comme ayant simplement besoin de supervision, ne va pas de soi. Il y a tout un spectre de mesures qui peuvent être utilisées et, ce faisant, qui peuvent atténuer l'écart entre les catégories extrêmes d'infracteurs. La tendance duale suppose que le contrôle pénal, en plus de qualifier et classifier les sujets de son action, les gère par deux voies extrêmes, l'une " soft » l'autre « bard », délaissant ainsi l'usage des voies médianes. C'est selon cette acception que l'expression « tendance duale » sera utilisée dans le cadre de cet article.

$\mathrm{Si}$, de l'avis de Cohen (1985), la tendance duale, prise dans un sens plus large, est demeurée centrale dans le système de contrôle social

9. « Put crudely, this bifurcation is between, on the one hand, the so-called "really serious offender" for whom very though measures are typically advocated; and, on the other hand, the "ordinary" offender for whom, it is felt, we can afford to take a much more lenient line " (Bottoms, 1977 : 88).

10. "At the same time that there has been a liberal "hands off" push toward decarceration and diversion, toward using the least severe option, there has arisen in relation to the penitentiary an increased sense that it contains the uncontainable, the dangerous. This has resulted in a "hands on" push within prisons - prisons within prisons, including protective custody, psychiatrics units, special handling units, administrative dissociation, and punitive dissociation, as well as arguments for administrative devices to keep the "dangerous" there (sugh as via "gating") even when previous administrative practice and the law suggest otherwise. So the carceral continuum stretches very deeply inside the prison and very widely into the outside community, according to an administrative model of who is more or less dangerous, more or less free » (Ericson, $1987: 24)$. 
depuis ses débuts, le critère à partir duquel elle s'effectue, lui, a changé : « All that has changed over the last century is the basis of binary classification. It used to be moral character, sometimes it was treatability or security risk, now it tends to be dangerousness » (Cohen, 1985 : 19945). Que la base de la classification binaire en 1985 soit ou non la dangerosité n'est pas ce qui intéresse au premier chef dans ce passage. Ce qu'il est nécessaire de faire ressortir ici, c'est le fait que la tendance duale peut se matérialiser à partir d'une diversité de critères de distinction. Les modèles assurantiel et prudentiel suggèrent que la gestion des risques est devenue la grille de lecture à travers laquelle on pense le problème de la criminalité et celui de la peine. On verra, dans la section qui suit, que cette lunette colore fortement la tendance duale observée dans la politique fédérale de mise en liberté sous condition.

\section{Historique des règles générales d'admissibilité à la mise en liberté sous condition au canada de 1899 à 1997}

À partir de 1978 et jusqu'au dernières modifications législatives, on verra se dessiner dans les textes de lois une distinction persistante entre deux catégories d'infracteurs. D'un côté, une partie des détenus deviendra admissible de plus en plus rapidement à l'une ou l'autre des formes de mises en liberté sous condition alors que de l'autre côté, un groupe d'infracteurs verra s'allonger la portion de la peine à purger avant la mise en liberté. Il faut voir aussi que le risque de récidive postulé devient, au fil du temps, un élément central dans l'admissibilité à la mise en liberté sous condition.

Pour les fins de la démonstration qui suit, seules les règles générales d'admissibilité à la mise en liberté sous condition seront examinées. Les règles d'admissibilité des infracteurs condamnés à perpétuité et à une sentence indéterminée sont donc exclues. De plus, par souci de brièveté, seuls les principaux changements survenus seront soulignés dans le corps du texte et dans le tableau 1.

L'origine de la mise en liberté sous condition au Canada remonte à $1899^{11}$. À cette époque, le texte de loi ne prévoyait aucune période à purger à l'intérieur des murs ni une admissibilité différentielle selon l'acte imputé aux détenus. Toutefois, dans la pratique, la règle était que

11. An Act to provide for the Conditional Liberation of Penitentiary Convicts, S.C. 1899, c.49. 
TABLEAU 1

Tableau synoptique des changements apportés à la règle générale d'admissibilité à la mise en liberté sous condition au Canada de 1899 à 1997

\begin{tabular}{|c|c|c|c|}
\hline Année & Semi-liberté & Lib. conditionnelle totale & Libération d'office \\
\hline 1899 & & $1 / 2$ de la sentence & \\
\hline 1960 & & $\begin{array}{l}1 / 3 \text { ou } 4 \text { ans (la moindre), } \\
\text { min. } 1 \text { an }\end{array}$ & \\
\hline 1964 & & $\begin{array}{l}1 / 3 \text { ou } 4 \text { ans (la moindre), } \\
\text { min. } 9 \text { mois }\end{array}$ & \\
\hline 1970 & 1 an avant l.c. * & $\begin{array}{l}1 / 3 \text { ou } 4 \text { ans (la moindre), } \\
\text { min. } 9 \text { mois }\end{array}$ & $2 / 3$ de la sentence \\
\hline 1973 & 1 an avant l.c. & $\begin{array}{l}1 / 3 \text { ou } 7 \text { ans (la moindre), } \\
\text { min. } 9 \text { mois }\end{array}$ & $2 / 3$ de la sentence \\
\hline 1978 & $\begin{array}{l}12 \text { ans et plus : } \\
2 \text { ans avant l.c. } \\
2-12 \text { ans : } 1 / 2 \text { avant } \\
\text { l.c. min. } 6 \text { mois (la } \\
\text { plus longue) }\end{array}$ & $\begin{array}{l}\text { Violents : récidivistes } \\
\text { violents } 1 / 2 \text { ou } 7 \text { ans } \\
\text { (la moindre) } \\
\text { Autres : } 1 / 3 \text { ou } 7 \text { ans } \\
\text { (la moindre), min. } 9 \text { mois }\end{array}$ & $2 / 3$ de la sentence \\
\hline 1986 & $\begin{array}{l}12 \text { ans et plus : } \\
2 \text { ans avant l.c. } \\
2-12 \text { ans : } 1 / 2 \text { avant } \\
\text { l.c. min. } 6 \text { mois (la } \\
\text { plus longue) }\end{array}$ & $\begin{array}{l}\text { Condamnés à } 3 \text { ans ou } \\
\text { moins : Procédure accélérée } \\
\text { Délit violent : récidivistes } \\
\text { violents de } 1 / 2 \text { ou } 7 \text { ans } \\
\text { (la moindre) } \\
\text { Autres : } 1 / 3 \text { ou } 7 \text { ans } \\
\text { (la moindre), min. } 9 \text { mois }\end{array}$ & $\begin{array}{l}\text { Délit violent : } \\
\text { possibilité de conditions } \\
\text { de résidence ou } \\
\text { non admissibles } \\
\text { Autres : } 2 / 3 \text { de la } \\
\text { sentence }\end{array}$ \\
\hline 1992 & 6 mois avant l.c. & $\begin{array}{l}\text { Premier terme de } \\
\text { pénitencier et infraction } \\
\text { autre que délit violent ou } \\
\text { lié à la drogue : Procédure } \\
\text { accélérée } \\
\text { Délit violent ou lié à la } \\
\text { drogue : selon la } \\
\text { détermination judiciaire }{ }^{* *} \text { : } \\
\text { possibilité de } 1 / 2 \text { ou } 10 \text { ans } \\
\text { (la moindre) } \\
\text { Autres : } 1 / 3 \text { ou } 7 \text { ans (la } \\
\text { moindre), min. } 9 \text { mois }\end{array}$ & $\begin{array}{l}\text { Délit violent ou lié à la } \\
\text { drogue : possibilité de } \\
\text { conditions de résidence } \\
\text { ou non admissibles } \\
\text { Autres : } 2 / 3 \text { de la } \\
\text { sentence }\end{array}$ \\
\hline
\end{tabular}


TABLEA U 1

Tableau synoptique des changements apportés à la règle générale d'admissibilité à la mise en liberté sous condition au Canada de 1899 à 1997 (suite)

\begin{tabular}{|c|c|c|c|}
\hline Année & Semi-liberté & Lib. conditionnelle totale & Libération d'office \\
\hline 1997 & $\begin{array}{l}\text { Premier terme de } \\
\text { pénitencier et } \\
\text { infraction autre que } \\
\text { délit violent ou lié à } \\
\text { la drogue : } 1 / 6 \text { ou } 6 \\
\text { mois (la plus longue) } \\
\text { Autres : } 6 \text { mois avant } \\
\text { l.c. }\end{array}$ & $\begin{array}{l}\text { Premier terme de } \\
\text { pénitencier et infraction } \\
\text { autre que délit violent ou } \\
\text { lié à la drogue : Procédure } \\
\text { accélérée } \\
\text { Délit violent ou lié à la } \\
\text { drogue : selon la } \\
\text { détermination judiciaire**: } \\
\text { possibilité de } 1 / 2 \text { ou } 10 \text { ans } \\
\text { (la moindre) } \\
\text { Autres : } 1 / 3 \text { ou } 7 \text { ans (la } \\
\text { moindre), min. } 9 \text { mois }\end{array}$ & $\begin{array}{l}\text { Délit violent ou lié à la } \\
\text { drogue : possibilité de } \\
\text { conditions de résidence } \\
\text { ou non admissibles } \\
\text { Autres : } 2 / 3 \text { de } \\
\text { la sentence }\end{array}$ \\
\hline
\end{tabular}

$\star$ Libération conditionnelle totale

${ }^{\star \star}$ C'est le tribunal qui prend la décision d'imposer l'incarcération pour la moitié de la peine ou dix ans avant la mise en liberté sous condition. Dans la pratique, il est rare que le tribunal formule cette exigence.

les détenus devaient passer au moins la moitié de leur peine au pénitencier (Fauteux, 1956 : 66).

Même si le nombre des libérés croît progressivement, ce n'est véritablement qu'avec l'instauration des recommandations du comité Fauteux en 1959, comité chargé d'étudier la question de la mise en liberté sous condition, que cette dernière se popularise. Les plus importantes recommandations du rapport Fauteux se trouvent concrétisées par l'adoption d'une loi qui entre en vigueur le 15 février $1959^{12}$. La loi instaure la Commission nationale des libérations conditionnelles. La règle générale est modifiée à l'effet qu'un détenu doit purger le tiers de sa sentence ou quatre ans, la moindre des deux périodes, mais un minimum d'un an doit être passé en détention ${ }^{13}$. En 1964, le minimum à purger passe d'un an de détention à neuf mois ${ }^{14}$.

C'est en 1970 que surviennent d'autres changements d'importance. En effet, une nouvelle forme de mise en liberté sous condition entre en

12. Loi sur la libération conditionnelle de détenus, S.C. 1959, c.38.

13. C.P. $1960-681$.

14. C.P. DORS/64-1827. 
vigueur : la libération d'office ${ }^{15}$. Contrairement au cas de la libération conditionnelle, la libération d'office est automatique. Cette mesure exige que la remise de peine soit passée sous surveillance. La remise de peine est un mécanisme qui permet aux détenus de soustraire un jour à leur sentence pour chaque période de deux jours purgés. La remise de peine compte donc pour un maximum du tiers de la sentence ${ }^{16}$. Par exemple, jusqu'en 1970, un détenu ayant reçu une sentence de 9 ans et à qui les autorités n'ont pas accordé de libération conditionnelle totale peut donc sortir du pénitencier après 6 ans de confinement compte tenu des jours de remise de peine auxquels il a droit. À partir de l'instauration de la libération d'office toutefois, le détenu de notre exemple devra passer de la sixième à la neuvième année de sa peine, non pas libre tel qu'il l'aurait été avant cette loi, mais sous surveillance dans la communauté ${ }^{17}$. Le même texte de loi instaure aussi la semi-liberté ${ }^{18}$. Les détenus en semi-liberté sont libres le jour mais doivent retourner tous les soirs au pénitencier ou dans un établissement prévu à cet effet. En 1970, les détenus y sont admissibles, en règle générale, un an avant la fin de leur période d'inadmissibilité à la libération conditionnelle totale, c'est donc dire un an avant le tiers de leur peine.

Les modifications suivantes surviennent en $1973^{19}$. On augmente le minimum à purger avant la libération conditionnelle totale. En effet, la règle générale, du tiers ou quatre ans qu'elle était, passe à un tiers ou sept ans. Avant comme après, c'est la moindre des deux périodes qui est retenue. Ce changement aura un impact sur les détenus dont la sentence a été fixée à plus de douze ans ; ceux-ci verront leur période d'inadmissibilité à la libération conditionnelle s'allonger.

L'année 1978 marque le début de la tendance duale en ce qu'elle crée le « hard end et rallonge la période d'inadmissibilité pour cette catégorie. En effet, le nouvel article 8 des règlements instaure une distinction

15. Nous utilisons ici l'appellation actuelle. Cette forme de mise en liberté s'est appelée « surveillance obligatoire » de 1970 à 1985, « liberté surveillée » de 1986 à 1992 et « libération d'office » depuis.

16. En fait, la remise de peine est plus complexe, car il y a une partie des jours remis qui est une remise « méritée » et une autre partie qui est statutaire, obligatoire. Cette distinction a été abolie plus tard.

17. Loi de 1968-69 modifiant le droit pénal, S.C. 1969, c.38.

18. Nous utilisons ici l'appellation actuelle. Cette forme de mise en liberté s'est appelée libération conditionnelle de jour de 1970 à 1985 et semi-liberté depuis.

19. C.P.DORS/73-298. 
qui perdurera dans les lois subséquentes c'est-à-dire la frontière entre ce que nous appellerons les « délinquants violents » et les autres :

8. (1) Sous réserve du paragraphe (2), lorsqu'un détenu doit purger une peine d'emprisonnement de cinq ans ou plus pour avoir commis une infraction le rendant passible d'un emprisonnement de dix ans ou plus, infraction qui

a) a mis gravement en danger la vie ou la sécurité d'autrui

b) a entraîné des sévices graves à autrui, ou

c) a causé des dommages psychologiques importants à autrui,

la partie de la peine d'emprisonnement que le détenu doit purger avant que la libération conditionnelle totale puisse lui être accordée, est la moitié de la peine d'emprisonnement imposée ou sept ans, suivant la période la plus courte. (DORS/78-428) (les caractères gras sont de nous).

Cependant, le paragraphe (2) vient restreindre grandement l'application de cette disposition en ce qu'elle n'est destinée qu'aux détenus qui, dans les dix dernières années, ont déjà purgé une peine pour un délit répondant à l'une des trois caractéristiques mentionnées au paragraphe un. Cette classification des infracteurs, et la tendance duale qui s'en suit, opère donc sur la base de la violence des infractions commises et de la récidive des infracteurs.

Les règlements refondus de 1978 sont aussi l'occasion de revoir les périodes d'inadmissibilité à la semi-liberté. Alors qu'en 1970, la semiliberté est possible un an avant la libération conditionnelle totale, des distinctions s'établissent en 1978. En effet, les infracteurs condamnés à douze ans et plus y sont admissibles une année plus tôt (deux ans avant le tiers ou deux ans avant sept ans, la moindre des périodes) alors que les détenus purgeant une peine de deux à douze ans sont admissibles à la semi-liberté à la moitié de leur période d'inadmissibilité à la libération conditionnelle totale (la moitié du tiers ou la moitié de sept ans, la moindre des périodes) ou six mois, la plus longue de ces périodes étant retenue.

En 1986, deux projets de loi importants viennent modifier la législation sur les libérations conditionnelles et renforcer la tendance duale instaurée en 1978. Le premier, le projet de loi C-67, porte sur le maintien en incarcération. À partir de ce moment, la CNLC peut refuser d'accorder la liberté surveillée aux détenus qui ont commis des infractions de violence, liberté qui était jusqu'alors acquise par l'accumulation de la remise de peine. De plus, dans les cas où la liberté surveillée est accordée, des conditions de résidence peuvent être imposées, autrement dit la Commission peut désormais exiger d'un détenu qu'il purge le 
dernier tiers de sa sentence sinon au pénitencier, du moins dans un établissement résidentiel communautaire. La décision de maintien en incarcération est prise quand trois conditions sont réunies :

a) le détenu purge une peine d'emprisonnement qui comprend une peine imposée à la suite d'une mise en accusation pour une infraction prévue à l'annexe ;

b) l'infraction a causé la mort ou un tort considérable ;

c) il existe des motifs raisonnables de croire qu'il commettra vraisemblablement, avant l'expiration légale de sa peine, une infraction telle que celle visée à l'alinéa b) (Loi modifiant la Loi sur la libération conditionnelle et la Loi sur les pénitenciers, L. R. C. 1985 , c. $34,2^{\mathrm{e}}$ supplément) (les caractères gras sont de nous).

Les infractions, prévues à l'annexe, qui rendent les détenus passibles d'un maintien en incarcération sont au nombre de 28 et comprennent des délits tels les bris de prisons, les crimes d'incendies, les infractions d'ordre sexuel mais surtout des infractions impliquant de la violence (homicide, voies de fait, vol qualifié, etc.). Soulignons le fait qu'une des conditions du maintien en incarcération est la prévision d'un comportement. Ainsi, la tendance duale change de critère : elle n'opère plus seulement sur la base de la violence de l'infraction passée comme en 1978, mais aussi sur la violence de l'infraction appréhendée, la récidive virtuelle. Ici, la référence aux théories classiques de la peine bascule. Alors que le système pénal canadien s'est surtout basé sur le principe selon lequel on ne punissait l'infracteur que pour le crime dont on le reconnaît coupable, on délaisse momentanément ce principe pour mettre de l'avant une mesure de neutralisation sélective basée sur la prédiction du comportement.

Si d'un côté le projet de loi C-67 resserre le contrôle sur les détenus dits violents, le projet de loi C-68 accélère le processus de remise en liberté des autres. À partir de 1986, afin de permettre aux détenus qui purgent une peine de trois ans ou moins de bénéficier de leur remise en liberté dès leur admissibilité, la CNLC statue sur la libération conditionnelle totale d'un détenu au moment de l'examen du dossier en semi-liberté. Ce faisant, les délais administratifs n'empêchent plus les détenus qui ont une courte sentence d'être en libération conditionnelle totale aussitôt que la loi les y autorise ${ }^{20}$.

Les dispositions de 1986 confirment donc une tendance amorcée en 1978, le traitement différentiel de deux catégories d'infracteurs, ceux qui

20. DORS/86-915, art. $14.1(2)$ 
sont réputés violents et les autres. La nouvelle loi transforme aussi le critère de distinction entre ces deux catégories d'infracteurs en ajoutant la récidive virtuelle à la violence de l'acte passé. C'est toutefois en 1992 que ce principe de tendance duale connaît sa pleine affirmation avec la Loi régissant le système correctionnel, la mise en liberté sous condition et le maintien en incarcération, et portant création du bureau de l'enquêteur correctionnel (S. C., 1992, c. 20).

En vertu de cette loi, la semi-liberté est retardée, pour toutes les catégories de détenus, à six mois avant l'admissibilité à la libération conditionnelle totale. Cette dernière est aussi passablement revue. La procédure accélérée est maintenant destinée aux infracteurs qui en sont à leur premier terme au pénitencier et qui n'ont pas commis d'infractions visées aux annexes ; il y a pour eux une présomption de libération si les commissaires n'ont aucune raison de croire que les individus commettront une infraction violente avant l'échéance de leur terme. La loi de 1992 vient donc redéfinir la branche «soft» de la tendance duale en fonction des antécédents mais aussi de la probabilité de récidive violente.

Avec cette même loi, l'annexe des infractions violentes instaurée en 1986 s'est « enrichie » de 14 nouvelles infractions (principalement des infractions sexuelles à l'endroit des enfants) et d'une deuxième annexe comprenant 11 infractions reliées à la drogue (trafic, culture, recyclage des produits de la criminalité, etc.). Ces listes identifient les crimes en vertu desquels un juge peut imposer au détenu qu'il purge la moitié de sa peine ou dix ans, plutôt que sept ans comme auparavant, la moindre des deux périodes étant retenue. Ce sont aussi les infractions énumérées dans ces annexes qui rendent les détenus passibles de conditions de résidence pendant leur libération d'office ou d'un maintien en incarcération jusqu'à la fin de leur peine ${ }^{21}$. Il semble donc que la branche «dure » de la tendance duale se soit enrichie de nouvelles catégories d'infracteurs « ̀̀ risques ».

Les dernières modifications à la règle d'admissibilité sont apportées par la loi C-17 de $1997^{22}$. Le changement principal induit par la loi est d'avancer la date d'admissibilité à la semi-liberté pour une catégorie de

21. Dans les années suivantes, les annexes seront modifiées pour inclure de nouvelles infractions (conduite avec facultés affaiblies, conduite dangereuse et harcèlement criminel [L.C. 1995, c.42], les infractions commises avec de fausses armes à feu [L.C. 1995, c.39]) ou encore pour respecter les changements apportés à d'autres lois, notamment à la Loi réglementant certaines drogues et autres substances.

22. Loi modifiant le Code criminel (délinquant présentant un risque élevé de récidive), la Loi sur le système correctionnel et la mise en liberté sous condition. La Loi sur le casier judiciaire, la Loi sur les prisons et les maisons de correction et la Loi sur le ministère du Solliciteur général, 1997, ch. 17, art.21 (1). 
détenus. En effet, les infracteurs qui en sont à leur premier terme au pénitencier et qui n'ont pas commis d'infractions énumérées dans les annexes peuvent être admissibles à la semi-liberté au $1 / 6$ de leur peine plutôt que seulement 6 mois avant la libération conditionnelle totale comme auparavant. La branche «soft » de la tendance duale se fait donc plus « soft».

Bref, d'après les transformations des lois et des règlements, particulièrement à partir de 1978, ce qui semble se dessiner, c'est une logique duale dans les politiques de mise en liberté sous condition. À partir de 1986, cette division s'opère entre autres sur la base du risque appréhendé, critère qui est confirmé en 1992. Ainsi, d'un côté, on tente de remettre en liberté le plus tôt possible des infracteurs que l'on juge ne représenter qu'un faible risque pour la société, de l'autre, on prend des dispositions afin de prolonger l'incarcération des infracteurs qui ont commis ou dont on croit qu'ils commettront, à leur sortie, des infractions graves.

Cette tendance duale observée dans les textes de loi se produit sur un fond idéel particulier. Afin de saisir le sens des changements de la politique de mise en liberté sous condition, une tentative sera donc faite pour comprendre ce qui anime les discours officiels et politiques à deux époques privilégiées : 1956-1960 qui correspond à l'instauration de la CNLC et 1988-1992 qui renvoie au processus ayant donné lieu à la Loi sur le système correctionnel et la mise en liberté sous condition. Comme il a été dit en introduction, bien que la tendance duale ne se manifeste que plus tard, l'examen de la première période se justifie par son effet de repoussoir, de point de comparaison à la deuxième période où la tendance duale s'affirme. Les deux sections suivantes, s'intéressant chacune à une époque, cherchent à saisir la façon dont la politique de mise en liberté sous condition a été dépeinte dans les discours officiel et politique, et comment on a présenté le système correctionnel, la mesure de mise en liberté, les infracteurs, etc. Ce contraste entre deux périodes montrera le changement de rationalité dans les discours et la façon dont la tendance duale s'y insère.

\section{La gestion par la réhabilitation 1956-1960}

L'historique de la mise en liberté sous condition le soulignait, c'est avec la concrétisation des recommandations du rapport Fauteux (1956), notamment l'instauration de la CNLC, que la mise en liberté sous condition devient une façon généralisée de sortir de la détention et prend ainsi la place qu'on lui connaît aujourd'hui dans le processus correctionnel. 
Dans le rapport Fauteux (1956), il est clair que les experts croient alors fermement que cette mesure est un outil clef dans la tâche du système correctionnel. On doit comprendre que la mise en liberté sous condition se prête bien à la définition du problème de la criminalité que l'on se fait à cette époque : «On peut dire que ceux qui enfreignent le droit criminel ont été «avariés» dans leur jeunesse [...] [ils] se sont écartés du droit chemin tracé par la loi » (Fauteux, 1956 : 76).

Suivant cette conception du problème de la criminalité, le rapport donne un second souffle, "thérapeutique ", aux libérations conditionnelles qui doivent dès lors viser deux principaux buts : la correction des infracteurs, premier objectif répondant inévitablement au second, et la sécurité de la collectivité. En effet, « chacun des éléments du système correctionnel a un rôle important et quelquefois vital à jouer. Chacun doit jouer son rôle à la lumière du principe fondamental du système correctionnel, qui est la correction de l'individu » (Fauteux, $1956: 5$ ). En « corrigeant» les détenus, on se trouve par le fait même à protéger le plus grand nombre :

La libération conditionnelle vise à assurer, dans la plus grande mesure possible, la sécurité de la collectivité aussi bien que le bien-être du particulier libéré. Ces deux objets sont inséparablement liés, parce que la sécurité du particulier dépend de la réforme du délinquant. Si ce dernier ne s'amende pas et commet de nouveaux délits à sa libération, la collectivité se trouve menacée. Pour cette raison, elle a un intérêt et une responsabilité directs dans l'avenir du détenu libéré. Si la libération conditionnelle réussit à celui-ci à mieux se réadapter à la vie avec ses semblables, elle contribue alors au bien-être de tous (Fauteux, 1956 : 55).

Dans ce contexte, la libération conditionnelle est définie comme : « [...] une étape logique dans la réforme et la réadaptation d'une personne qui a été trouvée coupable d'un délit [...] » (Fauteux, 1956 : 54). «Cette libération devrait être interprétée comme le résultat naturel, voire inévitable, [d'un séjour en prison où le détenu] devient mûr pour la libération » (Fauteux, 1956 : 56-57). La mise en liberté sous condition est donc une étape de transition qui conjugue à la fois la fonction de contrôle et la fonction d'assistance.

Dans l'arène politique, la mise en liberté sous condition est bien accueillie, même par l’opposition. Un régime structuré, centralisé et généralisé de mise en liberté bien campée dans une philosophie progressive de correction a tout pour plaire. En effet, l'expansion de la politique des libérations conditionnelles semble toute désignée pour combattre les deux principales failles du système d'alors soit son coût élevé et son impuissance à contrecarrer la récidive (Débats, 1956, $1: 433 ; 7$ : 7742). 
Tout comme les experts qui ont rédigé le rapport, les hommes politiques sont d'avis qu'il faut faire prendre un tournant décisif aux politiques pénales (Débats, 1959, $4: 5174$ ). Cette pénologie « moderne » est sous-tendue par des conceptions nouvelles concernant les délinquants et les mesures à employer à leur égard :

Ce sujet de la réhabilitation est, à mon avis, d'une extrême importance. Depuis quelques années, surtout à la suite des recherches poursuivies par la Commission Fauteux et des recommandations contenues dans le rapport soumis au cabinet par cette Commission, le gouvernement comme la société considèrent aujourd'hui le condamné sous une optique plus humanitaire. Les découvertes de Freud en psychanalyse, les travaux que ses disciples ont poursuivis en ce domaine et dans celui de la psychiatrie, ont depuis quelques années porté les hommes de loi et les législateurs à envisager d'une autre façon celui qui, autrefois, était qualifié catégoriquement de criminel. On pense aujourd'hui que le condamné n'est peut-être pas le seul responsable du crime qu'il a commis. La société, l'entourage, la famille, à un degré qu'il est impossible de préciser mais qui existe, ont également participé à la commission de ce crime. Heureusement, aujourd'hui, le condamné n'est plus considéré comme un criminel. Il demeure un être humain que la société se doit de réhabiliter (Débats, 1959, $4: 5133$ ).

La réadaptation sociale, le redressement, la réforme du délinquant, voilà justement à quoi se consacre la mesure :

Nous avons institué une nouvelle Commission des libérations conditionnelles qui examine le cas du point de vue psychologique et sociologique. Elle tient compte du délit que le détenu a commis et de toutes les circonstances; si nous pouvons réintégrer une personne dans la société, si nous pouvons sauver un Canadien par jour, nous accomplissons beaucoup dans ce domaine. Je trouve que la Commission nationale des libérations conditionnelles et ses dirigeants accomplissent un excellent travail à cet égard (Débats, 1961, $5: 5546$ ).

S'ajoute à cela le fait que les libérations conditionnelles semblent toutes désignées pour préserver la société de la récidive éventuelle des délinquants qui y sont soumis si l'on en croit les statistiques. En plus de faire l'unanimité du gouvernement et de l'opposition sur la question de l'efficacité quant à la réhabilitation, les libérations conditionnelles ont, croit-on, l'avantage de désengorger les institutions du surplus de détenus qu'on y retrouve. Cette retombée conjuguée avec le travail bénévole des sociétés post-pénales porte à croire que la mise en liberté sous condition sera des plus économique. Pour toutes ces raisons donc, la Loi relative à la libération conditionnelle de personnes purgeant des sentences d'emprisonnement entrera en vigueur en 1959. 
Bref, l'instauration de la Commission nationale des libérations conditionnelles et l'extension du régime de mise en liberté sous condition qui s'ensuivit se sont faites sur une toile de fond correctionnaliste. La mise en liberté sous condition s'inscrivait dans une compréhension de la déviance empreinte de déterminisme social. Le but de la mesure devenait dès lors la réhabilitation, objectif qui, s'il était atteint, assurerait automatiquement la protection du public. Dans ce contexte, la mise en liberté sous condition devenait une étape indispensable dans le processus correctionnel. Sur plusieurs de ces points - la section qui suit le démontrera - la fin des années 80 , où se développe la tendance duale, contraste avec les années 1956 à 1960.

\section{Le retour de la responsabilisation et la gestion des risques 1988-1992}

La fin des années 80 marque une époque fébrile en matière correctionnelle : plusieurs cas de récidives violentes par des libérés conditionnels font la manchette ; on termine aussi un débat sur le rétablissement de la peine de mort au Canada ; deux rapports qui proposent la restriction de l'usage de la mise en liberté sous condition sont déposés ${ }^{23}$. C'est dans ce contexte que le Comité permanent de la justice et du solliciteur général entreprend son enquête et dépose le rapport Daubney $(1988)^{24}$.

Ce document pose que « [1'] un des principaux problèmes à aborder de front est le degré d'inquiétude du public, inquiétude qui se transforme parfois en crainte et en panique » (Daubney, 1988 :3). Le manque de confiance des citoyens envers le système de justice est, d'après le rapport, attribuable aux « échecs » de la justice pénale qui n'est pas efficace afin de contrôler les criminels qu'elle remet en liberté. Le comité considère que certains échecs du système pénal et la crainte de la collectivité sont causés

23. La Commission Archambault (1987) argue pour une révision à la baisse des maxima fixés par le Code criminel et suggère que la mise en liberté sous condition soit une mesure réservée aux détenus condamnés à perpétuité. Le Groupe chargé de la révision du droit correctionnel (1987) propose quant à lui de maintenir le régime de mise en liberté sous condition mais de faire reposer les décisions de libération sur l'unique critère du risque encouru par la société.

24. Bien que cela ait pu être intéressant, les limites du présent article ne permettent pas d'aborder l'aspect de l'influence du contexte socio-politique des années 80 sur la transformation des politiques pénales. Comme il semblait impossible de parler brièvement du contexte socio-politique sans travestir la question délicate des liens entre le politique (vague de conservatisme), l'économique (la récession du début des années 80) et les questions pénales, l'option a été de réserver cette discussion pour un article ultérieur. 
par le manque de concordance entre la sentence prononcée et le temps purgé en incarcération : "À l'heure actuelle, le manque de confiance du public dans le système de justice criminelle est notamment attribuable à la période après laquelle le détenu devient admissible à un retour dans la société par mise en liberté sous condition, c'est-à-dire le tiers de la peine d'emprisonnement » (Daubney, $1988: 208$ ).

Un autre aspect qui fait consensus « [...] concerne la notion de responsabilité du délinquant, c'est-à-dire que si l'on enfreint la loi, on doit en assumer la responsabilité » (Daubney, $1988: 4$ ). Combinant cette idée de responsabilité et le but de protection des citoyens, le comité propose que l'on insiste sur le fait que la libération conditionnelle est un privilège. Fini le temps où la libération conditionnelle était une étape « naturelle et logique » du processus pénal. La libération conditionnelle doit se mériter. De plus, le fait de retarder l'admissibilité aux types de mise en liberté sous condition diminuerait l'écart entre la période réellement passée en prison et la longueur totale de la peine. Cela contribuerait, croit-on, à restaurer la confiance du public dans le système de justice pénal (Daubney, 1988 : 213).

Le simple report des dates de mise en liberté sous condition ne suffit pas selon le comité. Un resserrement est aussi nécessaire quant à la sélection des candidats. Celle-ci n'est possible que si les décideurs ont en main tous les renseignements nécessaires : "L'évaluation des risques et, partant, la prise de décisions dépendent de la qualité des renseignements fournis à la CNLC par le Service correctionnel du Canada » (Daubney, 1988 : 187). Un réaménagement organisationnel est proposé en ce sens. Les informations étant centralisées, la sélection des candidats et l'évaluation des risques qu'ils présentent se feront de façon plus éclairée. Cela permettra de mieux discriminer les délinquants dangereux des nondangereux, retardant ainsi la sortie des premiers (Daubney, 1988 : 192).

Sur la scène politique, à maintes reprises, on presse le Solliciteur général de présenter un projet de loi visant à réformer les libérations conditionnelles : «L'une des causes fondamentales de ces crimes violents est notre système judiciaire, qui permet des peines de prison beaucoup trop brèves ou qui accorde trop facilement la liberté conditionnelle [...]. Il faut rétablir la loi et l'ordre en ce pays » (Débats, 1991, 1 : 1272). Toutefois, ce n'est pas la voie de la loi et l'ordre que choisit précisément le gouvernement, puisqu'il cherche encore à réhabiliter les infracteurs : «Comme le Solliciteur général semble s'être engagé davantage envers la réadaptation des criminels, et c'est un aspect auquel nous croyons tous, il 
a semblé se désintéresser de ses responsabilités en tant que grand responsable du maintien de l'ordre au Canada » (Débats, 1991, 2 : 1903).

Les pressions populaires et politiques ainsi que le dépôt du rapport Daubney se solderont par le projet de loi C-36, Loi régissant le système correctionnel, la mise en liberté sous condition et l'incarcération, et portant création du bureau de l'enquêteur correctionnel. Ce projet sera présenté en première lecture le 8 octobre 1991 et, bien que critiqué par des dizaines de groupes de pressions, sera adopté quasiment tel quel en $1992^{25}$. Comme on l'a vu dans l'historique, cette loi prévoit retarder l'admissibilité à la semi-liberté. Celle-ci n'est possible que six mois avant l'admissibilité à la libération conditionnelle totale. La procédure de l'examen expéditif et une présomption de libération sont introduits pour les détenus qui en sont à leur premier séjour au pénitencier et qui ne sont pas incarcérés pour des infractions violentes ou liées à la drogue, donc pour aucun des articles prévus aux annexes I et II. Concernant ces annexes, la possibilité de maintien en incarcération est maintenue pour les infracteurs violents et étendue aux détenus incarcérés en vertu d'une infraction liée à la drogue.

En présentant la loi, le Solliciteur général, Doug Lewis, pose clairement que son but est la protection de la société :

Le projet de loi [...] accord[e] toute la priorité à la sécurité du public. Nous en avons fait le principe prépondérant de la loi. L'interprétation de cet important principe est très simple. Un détenu dont on envisage la mise en liberté et qui risque de mettre en danger la sécurité du public, n'est tout simplement pas libéré. Le gouvernement veut ainsi lancer un message à deux groupes de personnes. D'abord, il veut rassurer les membres du public qu'à partir de maintenant, ce sont eux et non les détenus qui auront le bénéfice du doute. Ensuite, il veut faire comprendre à tous ceux qui travaillent au sein du Régime de libération conditionnelle et du système pénitentiaire que ce sont les citoyens honnêtes qui passent en premier et que jamais la sécurité du public ne doit être compromise (Débats, 1991, 4 : 4430).

25. Malgré des critiques mordantes en Chambre, le projet de loi, intact, est transféré au Comité permanent. Là, on recevra quelque 80 groupes intéressés à la question. Les témoins sont très divers mais les témoignages vont presque tous dans le même sens : on ne croit pas que le projet de loi contribue à remplir son objectif, c'est-à-dire à protéger la société. Dans l'ensemble, les témoins, parce qu'ils le trouvent trop sévère ou pas assez, sont défavorables au texte proposé. Cela n'empêchera pas le projet de loi d'entrer en vigueur en 1992 en conservant l'esprit de départ et les règles d'admissibilité proposées en matière de mise en liberté sous condition. 
Encore ici, on reconnaît que, si réadaptation et protection de la société ne sont pas incompatibles, elles ne sont pas non plus synonymes : «Bien que nous ayons mis l'accent nécessaire sur la réadaptation, nous nous sommes aperçus que prenaient forme certaines lacunes graves donnant lieu à des résultats horribles et tragiques. Beaucoup a été fait pour les corriger, et nous pensons que ce projet de loi permet de rétablir l'équilibre » (Solliciteur général, Débats, 1991, $4: 4430$ ).

Dans la logique de la neutralisation sélective, on prétend que cette loi permettra de maintenir les détenus plus longtemps au pénitencier et qu'ainsi on améliorera la protection de la société. Dans ce contexte, la procédure d'examen expéditif « peut sembler étrange » (Débats, 1991, $4: 4431$ ), mais il vaut certainement mieux laisser les détenus non dangereux entretenir des liens avec leur proches et continuer leurs activités. De plus, « [c]ette façon de procéder dont on ne peut douter de la valeur sur le plan de la réadaptation, permettra de libérer près d'un milliard de dollars, somme affectée chaque année à l'incarcération, et d'axer davantage nos efforts sur la mise à l'écart des infracteurs violents et dangereux » (Solliciteur général, Débats, 1991, $4: 4431)$. On retrouve là un exemple de ce que Cohen (1985) a identifié comme étant une tendance duale dans le domaine du traitement, la réhabilitation ne valant que pour les cas légers, et une tendance duale dans le domaine des dépenses publiques, la redistribution des sommes d'argent « épargnées » s'effectuant du contrôle des petits infracteurs vers la gestion des infracteurs dangereux.

Avec la loi de 1992, il devient évident que la rationalité mise de l'avant n'est plus la « correction » des délinquants mais bien la protection du public. Les infracteurs sont maintenant représentés comme des individus responsables qui mettent en danger la sécurité des citoyens. La protection du public, clairement dissociée de la réhabilitation, passe dorénavant par un système de mise en liberté sous condition qui classifie les infracteurs selon qu'ils présentent peu ou beaucoup de risques et les gère en conséquence. On instaure, pour les premiers, une présomption de libération et un examen accéléré pour permettre leur réhabilitation. Par ailleurs, afin de protéger le public, on retient au pénitencier les détenus à hauts risques par le biais de l'allongement de la période d'inadmissibilité à la libération et par une nouvelle mesure, le maintien en incarcération, qui permet aux autorités de les détenir jusqu'à la fin de leur sentence. La réorganisation administrative des organismes chargés de la décision en matière de libération et de la surveillance des libérés se fait même ouvertement sur la base d'une meilleure gestion des risques. 
La comparaison entre les périodes 1956 -1960 et 1988-1992 révèle clairement le fait que les infracteurs déterminés font place aux infracteurs responsables. L'idéal de réhabilitation est remplacé par une volonté de protection croissante par une meilleure gestion des risques. De plus, alors qu'auparavant on se représentait la sécurité de la société et la réhabilitation des détenus comme indissociablement liées, elles sont maintenant isolées l'une de l'autre. En fait, la réhabilitation devient une préoccupation qu'on se permet pour certains infracteurs mais qu'on abandonne pour d'autres au profit d'un confinement prolongé. La mise en liberté sous condition n'est plus cette étape naturelle et logique dans le processus de réhabilitation mais bien le privilège des infracteurs à faible risque.

\section{Conclusion}

Le premier objectif que visait cet article était de démontrer que la législation concernant la mise en liberté sous condition au Canada est marquée progressivement, à partir de 1978, par une tendance duale, une inclinaison à punir de plus en plus sévèrement les vrais infracteurs, les « dangereux », alors qu'à l'inverse, les « petits » infracteurs sont traités avec une clémence croissante. Alors qu'il y a maintenant une présomption de libération, en semi-liberté, au sixième de la peine pour des infracteurs sans antécédents, les détenus ayant commis une infraction de violence ou liée à la drogue se voient imposer des mesures de plus en plus restrictives, aussi bien quant à l'allongement de la période d'inadmissibilité que quant à la libération conditionnelle totale, ou aux conditions de résidence pendant la libération d'office ou même au maintien en incarcération. Ainsi, si la mise en liberté sous condition est menacée d'attrition, cela ne toucherait qu'une partie des infracteurs, les délinquants à risques élevés. Pour le moment, ce qui semble représenter le mieux la tendance que prend la politique de mise en liberté sous condition est en fait l'image de la tendance duale. Bien qu'elle soit peu documentée, cette dernière est bel et bien une tendance qui fait sa place dans certaines politiques correctionnelles au Canada.

Un autre élément qui ressort de l'analyse du texte de loi sur la mise en liberté sous condition est le fait que la tendance duale peut opérer sur la base de plusieurs critères. En effet, la distinction entre les infracteurs de la branche «soft» et ceux de la branche « hard» ne se base pas uniquement sur les caractéristiques des crimes commis, la violence des 
infractions passées, mais de plus en plus sur les risques appréhendés, la récidive violente virtuelle.

Pour sa part, la transformation des discours politique et officiel sur la mise en liberté sous condition montre le rationnel en arrière plan des transformations de la loi. Ce dont on s'aperçoit, c'est que ces discours appuient la tendance duale, renforcent l'importance du critère de risque et la responsabilisation des infracteurs, tout en s'accompagnant d'une sévérité accrue : la volonté d'économiser dans la gestion des " petits » infracteurs pour réserver les fonds publics à la détention des infracteurs « à risques » ; l'utilisation d'une rationalité de protection de la société dans la gestion des infracteurs « dangereux » parallèlement à l'utilisation d'une rationalité de réhabilitation pour les " petits » infracteurs ; le discours sur la responsabilisation des infracteurs qui justifie la détention prolongée d'une partie d'entre eux ; la représentation de la mise en liberté sous condition comme un privilège pour les infracteurs à faibles risques; la réorganisation de la CNLC et du service de surveillance sur la base d'une meilleure communication, donc d'une meilleure gestion des risques; la promotion de la sécurité du public par la prédiction des risques, etc.

Plus globalement, les transformations que l'on observe dans la loi et le discours sur la mise en liberté sous condition au Canada ne sont pas étrangères aux caractéristiques du modèle de "nouvelle pénologie ». Feeley et Simon (1992) mettent de l'avant trois éléments de ce nouveau modèle : a) les nouveaux discours où les préoccupations pour la réhabilitation font place au langage de la probabilité et de la gestion du risque ; b) les nouveaux objectifs où la gestion du crime a remplacé l'élimination du crime ainsi que $\mathrm{c}$ ) les nouvelles techniques où la gestion des groupes a remplacé l'individualisation de la peine.

Quant au discours, il est vrai que celui de la mise en liberté sous condition n'a pas totalement évacué l'idée de réhabilitation. Il a tout au plus réduit celle-ci à un groupe d'infracteurs. En contrepartie, bien qu'il ne soit pas pleinement présent, l'intérêt pour la gestion des risques se profile avec de plus en plus d'insistance. Concernant les objectifs, si la mise en liberté sous condition cherchait auparavant à protéger la société en «sauvant » des Canadiens, maintenant, le même objectif de protection de la société veut être atteint par la détention prolongée de certains infracteurs. On ne cherche plus à éliminer le crime, mais plutôt à le contenir. Finalement, concernant les techniques, bien que l'on ne puisse dire si l'individualisation de la peine est toujours présente, on s'aperçoit que la politique de mise en liberté sous condition fait place de plus en 
plus à la gestion des groupes : la création des annexes I et II pour désigner les groupes d'infracteurs passibles de la mesure de maintien en incarcération; les infracteurs qui en sont à leur premier terme de pénitencier et qui n'ont pas commis d'infraction de violence ou liée à la drogue constituent le groupe pour lequel il y a la présomption de libération et l'examen accéléré.

Ainsi, les transformations récentes de la politique de mise en liberté sous condition laissent percevoir des signes selon lesquels les modèles assurantiel et prudentiel, au centre desquels on retrouve une préoccupation croissante pour la gestion des risques, font leur niche dans les politiques correctionnelles canadiennes.

\section{Références}

ARChambault, J. 1938. Rapport de la Commission royale d'enquête sur le système pénal du Canada, Ottawa : Imprimeur de la Reine.

ARCHAmbault, J. R. O. 1987. Réformer la sentence: une approche canadienne. Rapport de la Commission canadienne de la détermination de la peine, Ottawa : Approvisionnement et Services Canada.

BLANCHETTE, J. 1991. L'émergence de normes pénales en matière de piratage de logiciels. Une analyse des documents législatifs fédéraux de 1971 à 1981, Mémoire de maîtrise, École de criminologie, Université de Montréal.

Blumer, M. 1980. «Introduction » Pp. 1-9 in Social Research and Royal Commissions, sous la direction de M. Blumer. London : George Allen and Unwin.

Botтoms, A. 1977. «Reflection on the Renaissance of Dangerousness » The Howard Journal of Penology and Crime Prevention 16 (2) : 70-95.

Brodeur, J.-P. 1978. Les Commissions d'enquêtes publiques sur la police au Québec, Montréal : École de criminologie, Université de Montréal.

Brodeur, J.-P. 1990. « The Attrition of Parole » Revue Canadienne de Criminologie $32(3): 503-510$.

CANADA. 1956. Débats de la Chambre des Communes, 1, 364-365; 432-439; 1034-1035.

CANADA. 1956. Débats de la Chambre des Communes, 3, 2428-2433; 3280.

CANADA. 1956. Débats de la Chambre des Communes, 4, $3560 ; 4407$.

CANADA. 1956. Débats de la Chambre des Communes, 6, 6379-6380.

CANADA. 1956. Débats de la Chambre des Communes, 7, 7742-7745 ; 7762-7765.

CANADA. 1957. Débats de la Chambre des Communes, 1, 774-777 ; 913.

CANADA. 1957. Débats de la Chambre des Communes, 2, 1568-1569 ; 1620-1621.

Canada. 1957. Débats de la Chambre des Communes, 1, 130.

CANada. 1957. Débats de la Chambre des Communes, 3, 3256-3265. 
CANADA. 1958. Débats de la Chambre des Communes, 1, 1038.

CANADA. 1958. Débats de la Chambre des Communes, 2, 1120-1121 ; 1462-1463 ; 1923-1924; 2318-2321.

CANADA. 1958. Débats de la Chambre des Communes, 3, 3186-3196 ; 3356-3363 ; 3540-3543.

CANADA. 1958. Débats de la Chambre des Communes, 4, 3912-3917 ; 5003-5005.

CANADA. 1959. Débats de la Chambre des Communes, 1, 1052-1065.

CANaDA. 1959. Débats de la Chambre des Communes, 3, 2870 ; 3066.

CANADA. 1959. Débats de la Chambre des Communes, 4, 5132-5133 ; 5158-5159; 5174-5181; 5216-5217; 5222-5223.

CANADA. 1960. Débats de la Chambre des Communes, 5, 5542-5547 ; 5660-5661 ; 5760-5761.

CANADA. 1960. Débats de la Chambre des Communes, 6, 6116-6123.

CANadA. 1961. Débats de la Chambre des Communes, 6, 6766-6785 ; 6801-6805.

CANadA. 1988. Débats de la Chambre des Communes, $13366 ; 13763 ; 13847$; 13958 ; 14041-14042 ; 14181-14182 ; 14245 ; 14508 ; 15621 ; 16428 ; $16483 ; 16494 ; 16514 ; 16643 ; 16710-16712 ; 16743 ; 17093 ; 17962$; $18266 ; 18817 ; 19467-19468$.

Canada. 1989. Débats de la Chambre des Communes, 728 ; $3530 ; 3902 ; 4097$; 7001-7002; $7190 ; 8056$.

Canada. 1990. Débats de la Chambre des Communes, 9282; 10654-10655; 10877 ; $12166-12167$; 12447 ; 12727-12728; $15830 ; 17715$.

CANadA. 1991. Débats de la Chambre des Communes, $1-4,728 ; 1212 ; 1272$; 1903 1904 ; 2060-2061 ; 3303-3304 ; 4430-4449 ; 4473-4501 ; 4514-4541.

CANADA. SOlliciteur GÉNÉRAl. 1987. La Mise en liberté sous condition. Révision $d u$ droit correctionnel, Document de travail $\mathrm{n}^{\circ}$ 3, Ottawa: Secrétariat du ministère.

Carrière, P. et Silverstone, S. 1976. Le Processus de libération conditionnelle, Ottawa : Approvisionnement et services.

CASTEL, R. 1991. " From Dangerousness to Risk », Pp. 281-298 in The Foucault Effect. Studies in Governmentality. With Two Lectures by and an Interview with Michel Foucault, sous la direction de G. Burchell, C. Gordon et P. Miller . Chicago : University of Chicago Press.

Cavadino, M. et Dignan, J. 1992. The Penal System. An Introduction, London : Sage Publications.

Chapman, R. A. 1973. "Commissions in Policy-Making », Pp. 174-189 in The Role of Commissions in Policy Making, sous la direction de R. A. Chapman. London : George Allen and Unwin.

COHEN, S. 1985. Visions of Social Control, Cambridge : Polity Press.

Cole, D. P. et MANSON, A. 1990. Release from Imprisonment. The Law of Sentencing Parole and Judicial Review, Toronto : Carswell.

DAUBNEY, D. 1988. Des Responsabilités à assumer. Rapport du Comité permanent de la justice et du solliciteur général sur la détermination de la peine, la mise en liberté sous 
condition et d'autres aspects du système correctionnel, Ottawa: Approvisionnements et Services.

ERICSON, R. V. 1987. «The State and the Criminal Justice Reform », Pp. 20-37 in State Control: Criminal Justice Politics in Canada, sous la direction de R. S. Ratner et J. L. McMullan. Vancouver : University of British Columbia Press.

EWALD, F. 1991. "Insurance and Risk», Pp. 197-210 in The Foucault Effect. Studies in Governmentality. With Two Lectures by and an Interview with Michel Foucault, sous la direction de G. Burchell, C. Gordon, et P. Miller. Chicago : University of Chicago Press.

FAUTEUX, G. 1956. Rapport d'un comité institué pour faire enquête sur les principes et les méthodes suivis au Service des pardons du Ministère de la Justice du Canada, Ottawa : Imprimeur de la Reine.

Feeley, M. et Simon, J. 1992. "The New Penology: Notes on the Emerging Strategy of Corrections and its Implications »Criminology 30 (4) : 449-474.

FeELEY, M. et Simon, J. 1994. « Actuarial Justice: the Emerging New Criminal Law », Pp. 173-201 in The Futures of Criminology, sous la direction de D. Nelken. London : Sage Publications.

FranKS, C. E. S. 1987. The Parliament of Canada, Toronto: University of Toronto Press.

LUPTON, D. 1999. Risk, London : Routledge.

Normandeau, A. 1979. «Le Public et la violence : l'analyse des rapports des commissions d'enquête » Criminologie 12 (1) : 81-88.

O’Malley, P. 1992. «Risk, Power and Crime Prevention » Economy and Society 21 (3) : 252-275.

Peters, A. A. G. 1986. « Main Currents in Criminal Law Theory » in Criminal Law in Action, sous la direction de J. van Dijk, C. Haffmans, F. Ruter et J. Schutte. Arnhem : Gouda Quint.

Polvi, N. et Pease, K. 1991. "Parole and its Problems: A Canadian-English Comparison » The Howard Journal 30 (3) : 218-230.

PrATT, J. 1995. "Dangerousness, Risk and Technologies of Power» The Australian and New Zealand Journal of Criminology 28 (1):3-31.

REICHMAN, N. 1986. « Managing Crime Risks: Toward an Insurance Based Model of Social Control » Research in Law, Deviance and Social Control 8 : 151-172.

RoberT, D. 1994. L'Esprit et la lettre. Les libérations conditionnelles au Canada de 1956 à 1992, Mémoire de maîtrise, École de criminologie, Université de Montréal.

RoBIN, R. 1987. "L'Émergence du droit en commission parlementaire: la circulation de la parole publique et ses risques », Pp. 154-202 in Pour une approche critique du droit de la santé. Droit et matérialisation des politiques sociales, sous la direction de A. Lajoie, P. A. Molinari, P. Mulazzi, J.-M. Fecteau, R. Robin, C. Gosselin et J.-P. Ménard. Montréal : Les Presses de l'Université de Montréal.

SimON, J. 1987. "The Emergence of a Risk Society: Insurance, Law and the State » Socialist Review 95 : 61-89. 
SIMON, J. 1988. « The Ideological Effect of Actuarial Practices » Law and Society Review $22: 771-800$.

Simon. J. et Feeley, M. 1995. «True Crime: The New Penology and Public Discourse on Crime », Pp. 147-180 in Punishment and Social Control. Essays in Honor of Sheldon L. Messinger, sous la direction de T. G. Blomberg et S. Cohen. New York : Aldine de Gruyter. 